\title{
Simulation of Elastic Waves Propagation in 1D Stratified Porous Media for Low and High Frequency Domains
}

Igor Barbosa de Oliveira, Marcia Miranda Azeredo and Viatcheslav Ivanovich Priimenko, Laboratório de Engenharia e Exploração de Petróleo - LENEP / Universidade Estadual do Norte Fluminense Darcy Ribeiro - UENF

Copyright 2019, SBGf - Sociedade Brasileira de Geofísica

This paper was prepared for presentation during the $16^{\text {th }}$ International Congress of the Brazilian Geophysical Society held in Rio de Janeiro, Brazil, 19-22 August 2019.

Contents of this paper were reviewed by the Technical Committee of the $16^{\text {th }}$ International Congress of the Brazilian Geophysical Society and do not necessarily represent any position of the SBGf, its officers or members. Electronic reproduction or represent any position of the SBGf, its officers or members. Electronic reproduction of
storage of any part of this paper for commercial purposes without the written consent storage of any part of this paper for commercial
of the Brazilian Geophysical Society is prohibited.

\section{Abstract}

It was considered the low and high frequency domains for elastic waves propagation in 1D stratified porous media, at reservoir and laboratory scales, respectively. The general purpose was to simulate the poroelastic response, considering a mixed problem for the systems of Biot and Biot-JKD equations. Ursin formalism was used to construction of the solution. In addition, a dispersion and attenuation analysis of the waves propagated through a poroelastic medium was performed, relating frequency dependence to phase velocities and wave attenuation. An algorithm was created to construct the solution of the systems and a computational code was implemented to execute simulations. As expected by theory, at simulations the propagation of the slow compressive wave of Biot did not occur for low frequencies. However, for the high frequency domain, the inertial effects were predominant and the slow mode was activated. Moreover, during the simulations for high frequency domain the presence of the converted compressional fast-slow and also slow-fast wave was observed, where the elastic wave propagating in the porous medium converted its propagation mode by refracting or reflecting at an interface between two different poroelastic media.

\section{Introduction}

In this work a study of the classic Biot theory, developed in the middle of the 50s of the 20th century, is carried out, describing the propagation of elastic waves through a porous medium containing fluid. Both cases of low (BIOT, 1956a) and high (BIOT, 1956b) frequencies were considered.

The theory of poroelasticity was initiated through experimental observations made by DARCY (1856). The concept of porous media arose from the works of TERZAGHI (1923) in the area of soil mechanics, where the notion of effective pressure was introduced to describe the influence of the fluid that saturates the porous material in the quasi-static deformation of the soils. This theory assumes that the anelastic effects arise from viscous interaction between the fluid and the solid and predicts the existence of two compressive waves and one shear.
The fast compressional wave corresponds to the movement in which the displacement of the solid and the fluid are in phase, whereas the slow compressional wave is associated to the movement in which the displacements of the solid and fluid phases are not in phase.

For low frequencies, the medium does not support the slow wave. This becomes diffusive, since the effects of fluid viscosity dominate the inertial effects.

For high frequencies, the inertial effects are predominant and the slow mode is activated. Consequently, the amplitude of the wave field is attenuated due to energy losses in the presence of viscous fluid, as discussed further in the dispersion and attenuation analysis of the waves that propagates through a poroelastic medium.

The porous medium is composed of a solid phase and spaces between this solid phase, these spaces may contain fluids. An example of a natural porous medium is the rock oil reservoir.

In 1987, JOHNSON, KOPLIK and DASHEN (JKD) published a general expression for energy dissipation in the case of random pores, leading to the Biot-JKD model. This model is determined by the dynamic permeability, which results from the appearance of the viscous layer on the walls of the pores.

Usually the porous medium is treated as a fictitious continuous medium, so that it is possible to calculate for any point in the porous medium any desired physical property. Thus, when considering the continuous porous medium, it is assumed that there is an infinitesimal elemental volume representative of all the physical properties of the medium.

Poroelasticity theory

Theory of ordinary and partial differential equations

Ursin's formalism (1983)

Linear algebra

Numerical methods

Programming in MatLab

Figure 1: Theoretical bases of this work 
Recently, AZEREDO (2013), using the matrix method, has developed an algorithm capable of analytically and numerically solving the Biot poroelasticity equations that model the propagation of the wave in a three-dimensional medium, considering only the low frequency scheme. Following this line of research, MIRANDA (2016) created a mathematical-numerical algorithm for the analysis of the propagation of elastic waves in 3D porous media considering the effect of high temporal frequencies.

The theoretical bases of this work are based on poroelasticity theory, on the theory of ordinary and partial differential equations, on Ursin's formalism (1983), on linear algebra, numerical methods and programming in MatLab, as shown in Figure 1. The results shown in this expanded abstract are resulting from the master's thesis of OLIVEIRA (2018).

In this work are presented numerical simulations for the propagation of an elastic wave in saturated porous media. It is considered a one-dimensional analysis of horizontally stratified geological models, composed of homogeneous and isotropic layers. For this purpose two cases are considered, as mentioned previously: low and high frequencies, related to the reservoir and laboratory scale, respectively.

An algorithm was developed to construct the solutions of the systems of the Biot and Biot-JKD equations. Subsequently, a computer program was implemented to perform wave propagation simulations on 3-layer stratified 1D porous media.

The hypotheses considered in the development of this work are listed in Figure 2 below.

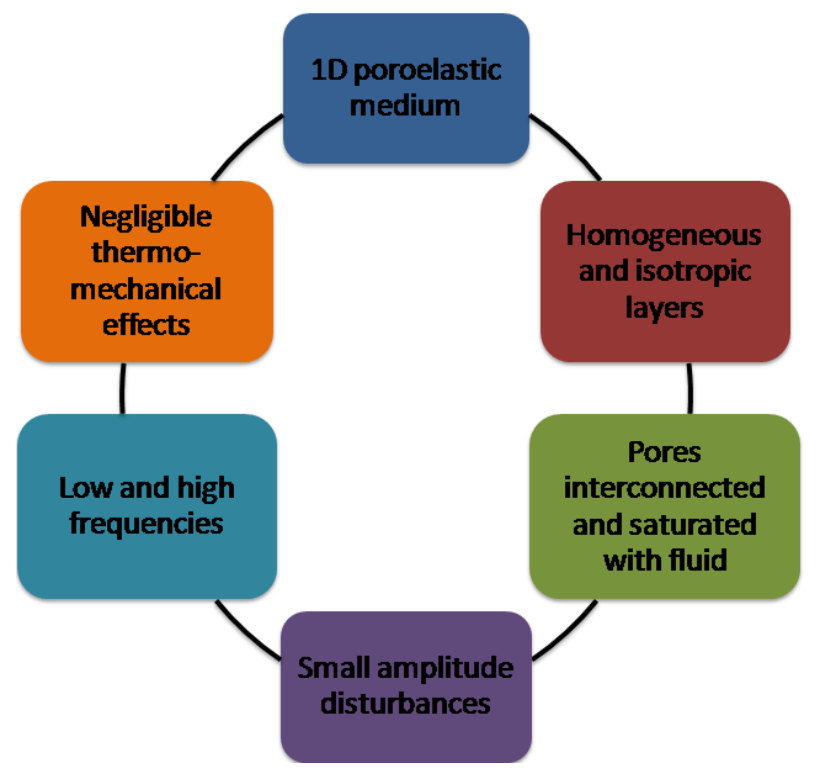

Figure 2: Hypotheses considered in the development of this work

The general purpose of this work was to simulate the poroelastic response of 1D stratified porous media, considering a mixed problem for the systems of the Biot and Biot-JKD equations and then perform a numericalanalytical analysis of the influence of variation of parameters to the problem's solution.

\section{Method}

The system of the Biot and Biot-JKD equations in the time domain for the one-dimensional case can be written through Equations (1) - (5) below.

$$
\begin{array}{ll}
\rho \frac{\partial v}{\partial t}+\rho_{f} \frac{\partial q}{\partial t}=\frac{\partial \sigma}{\partial z}+g & \\
\rho_{f} \frac{\partial v}{\partial t}+F_{e} \rho_{f} \frac{\partial q}{\partial t}=-\frac{\partial p_{f}}{\partial z}-\chi(t, q)+e & \\
\frac{\partial \sigma}{\partial t}=(\lambda+2 G) \frac{\partial v}{\partial z}+C M \frac{\partial q}{\partial z} & \\
\frac{\partial p_{f}}{\partial t}=-C M \frac{\partial v}{\partial z}-M \frac{\partial q}{\partial z} & , \text { Biot } \\
\chi(t, q)=\frac{\eta}{k}\left\{\begin{array}{ccc}
\int_{0}^{t} \frac{e^{-\Omega(t-\tau)}}{\sqrt{\pi(t-\tau)}}\left(\frac{\partial q(\tau)}{\partial \tau}+\Omega q(\tau)\right) d \tau & , \text { Biot }-J K D
\end{array}\right.
\end{array}
$$

where $\rho$ is the density of the medium, $\rho_{f}$ the density of the fluid, $F_{e}$ is the electrical factor of formation, $\lambda$ the Lamé parameter, $G$ the shear modulus, $C$ and $M$ are the Biot modules, $\eta$ is the viscosity of the fluid, $k$ is the permeability of the medium, $\Omega$ is a relaxation frequency, $g$ is the external force in the solid phase, $e$ the external force in the liquid phase, $v$ is the absolute displacement velocity of the matrix, $q$ is the rate of filtration of Darcy, $\sigma$ is the stress and $p_{f}$ the fluid pressure.

In this work, the poroelastic media considered are stratified and formed by homogeneous and isotropic layers, where each layer has constant physical properties in its interior, that is, such parameters are functions that are constant in parts, varying only according to depth $z$. Due to the stratification of the poroelastic media, there is the presence of discontinuities, that is, layer boundaries with different properties. The phenomenon that describes the diffraction, refraction and reflection of the waves is directly related to the presence of these interfaces. Figure 3 shows the boundary conditions and initial data.

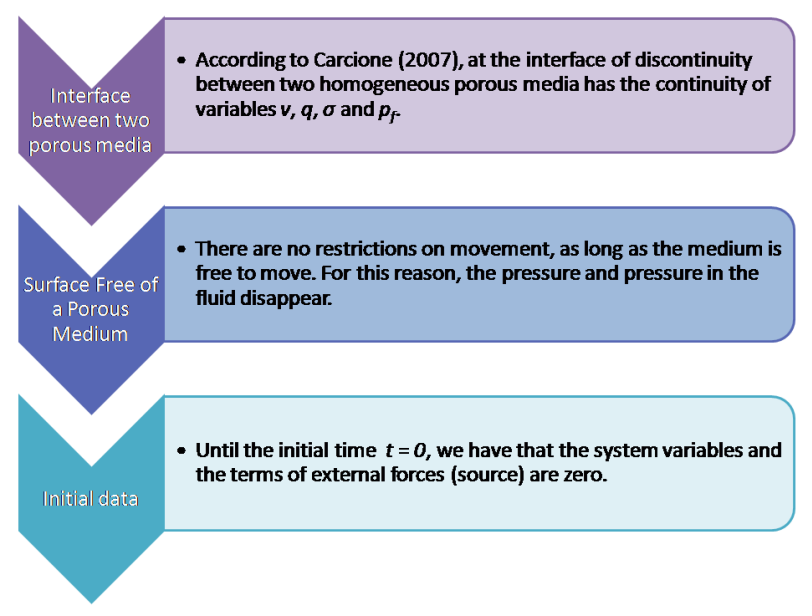

Figure 3: Boundary conditions and initial data

To transform the equations of the Biot and Biot-JKD systems from the time domain to the frequency domain $\omega$, the Fourier transform was used. 
Then, the dispersion and attenuation analysis of the waves propagated through a 1D poroelastic medium was performed, relating frequency dependence to phase velocities and wave attenuation. The velocity variation as a function of frequency is defined as the dispersion, which occurs due to the relative motion between the solid rock matrix and the fluid saturating the porous medium, since the attenuation is associated with the exponential decay rate of the wave amplitude in distance.

In conventional seismic surveys the frequency range used is between about 10 and $200 \mathrm{~Hz}$; in well profiling the frequency band is between a few hundred hertz up to 100 $\mathrm{kHz}$; in the laboratory high frequency waves are used, in the megahertz scale (GARCíA, 2008).

The boundary between the low frequency domain and the high frequency domain is determined when the viscous and inertial forces are similar and is defined as the critical frequency, $f_{C}$, (BLANC, 2013).

Figures 4-7 show the dispersion and attenuation curves corresponding to the Biot and Biot-JKD models. The physical properties of the porous medium and water were taken from BLANC (2013), while the properties of oils were taken from AL-BESHARAH (1987). Four different experiments were performed: (1) $100 \%$ porous medium saturated with water; (2) $100 \%$ porous medium saturated with light oil $\left(36^{\circ} \mathrm{API}\right) ;(3) 100 \%$ porous medium saturated with medium oil $\left(24.7^{\circ} \mathrm{API}\right)$ and (4) $100 \%$ porous medium saturated with heavy oil (14.8 $\mathrm{API})$.

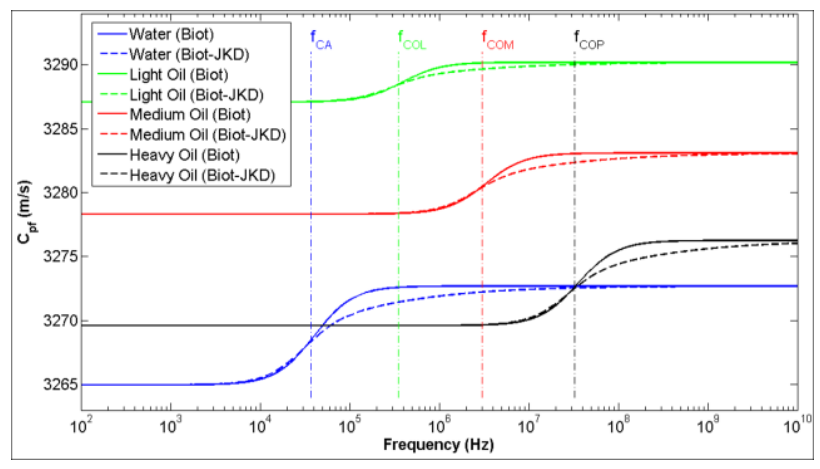

Figure 4: Fast wave phase velocity

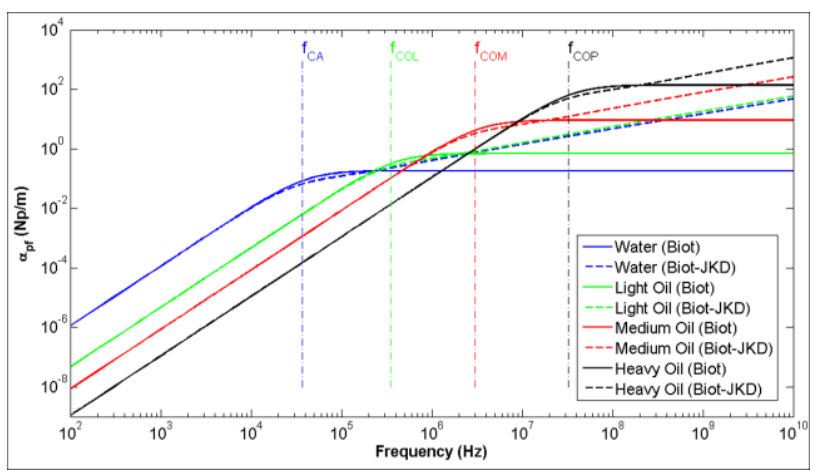

Figure 5: Fast wave attenuation curve

It is observed that higher the viscosity of the fluid, higher the critical frequency, that is, the higher the frequency of transition between the low and high frequency regimes.

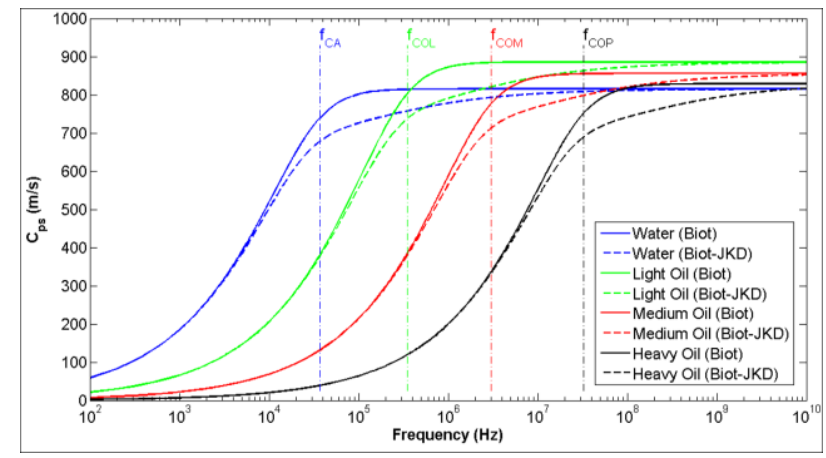

Figure 6: Slow wave phase velocity

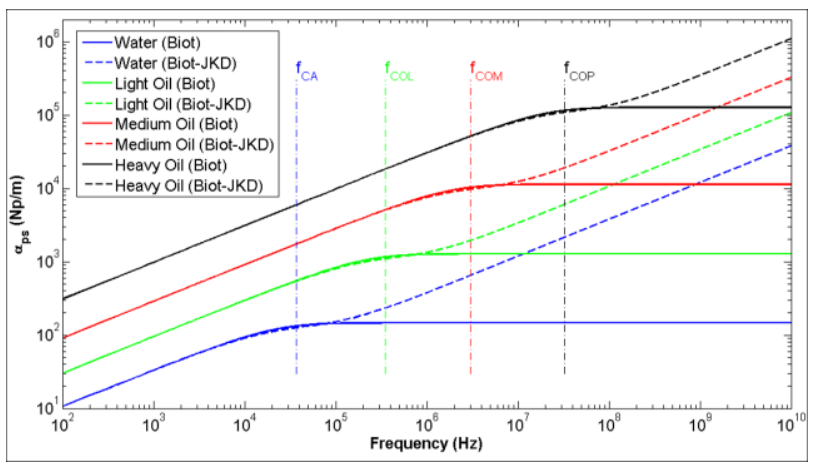

Figure 7: Slow wave attenuation curve

At low frequencies, the attenuation curves of Biot and Biot-JKD are very similar; which was already expected, since the frequency correction of the Biot-JKD model affects mainly the regime of the high frequencies. In addition, for low frequencies, the more viscous the fluid, lower the attenuation of the fast wave, and for very high frequencies, the opposite occurs. Comparing the graphs of attenuation of the fast wave with the slow wave it is noticed that the fast wave is little affected by frequency correction, whereas the slow wave is very affected.

Based on the ideas of Ursin (1983), the systems of the ordinary differential equations of Biot and Biot-JKD in the frequency domain were written in the matrix format. This development was able to decompose the analytical solution of the systems into two parts: upwards and downwards waves. After the calculation of the solution, the inverse Fourier transform was applied, obtaining the solution in the original domain. For more details see OLIVEIRA (2018).

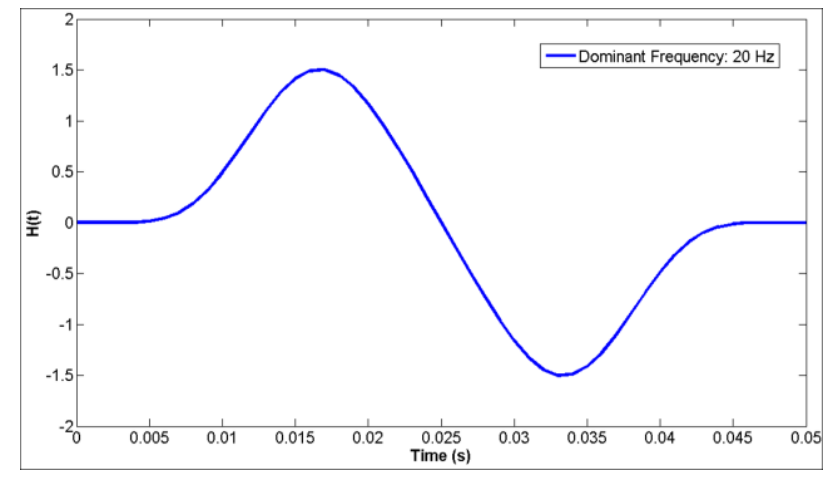

Figure 8: Source function - $H(t)$ 
Table 1: Physical properties of the stratified porous medium used in the simulations of elastic wave propagation

\begin{tabular}{|l|c|c|c|}
\hline Property & Unity & Layers 1\&3 & Layer 2 \\
\hline Density of fluid & $\mathrm{kg} / \mathrm{m}^{3}$ & 1040 & 1000 \\
\hline Viscosity of fluid & $\mathrm{Pa} . \mathrm{s}$ & $10^{-3}$ & $10^{-3}$ \\
\hline Density of solid & $\mathrm{kg} / \mathrm{m}^{3}$ & 2650 & 2644 \\
\hline Shear modulus & $\mathrm{Pa}$ & $1,85 \times 10^{9}$ & $7,04 \times 10^{9}$ \\
\hline Porosity & - & 0,3 & 0,2 \\
\hline Tortuosity & - & 2 & 2,04 \\
\hline Permeability & $\mathrm{m}^{2}$ & $1 \times 10^{-12}$ & $3,6 \times 10^{-13}$ \\
\hline Lamé parameter & $\mathrm{Pa}$ & $8,4 \times 10^{9}$ & $1,06 \times 10^{10}$ \\
\hline Biot modulus & $\mathrm{Pa}$ & $7,05 \times 10^{9}$ & $9,7 \times 10^{9}$ \\
\hline Biot modulus & - & 0,88 & 0,72 \\
\hline Viscous characteristic length & $\mathrm{m}$ & $7,3 \times 10^{-6}$ & $5,88 \times 10^{-6}$ \\
\hline
\end{tabular}

In order to simulate the propagation of elastic waves, the presence of a source represented by a function composed of truncated senoids (BLANC, 2013) was considered, see Figure 8. In addition, a 1D layered poroelastic medium was considered, with 3 layers. The physical properties of each layer are shown in Table 1.

\section{Results}

The results of the simulations are presented separately in relation to the frequency domain they belong to. First, the graphs of the absolute displacement velocity of the matrix for the Biot and Biot-JKD systems for low frequencies (reservoir scale) and then for high frequencies (laboratory scale) are displayed.

For the low frequency domain, a vertical punctual source with dominant frequency of $20 \mathrm{~Hz}$ was used, whereas a source with dominant frequency of $200 \mathrm{kHz}$ was used for the high frequency domain. The Figures 9 and 12 present the geological model and source-receiver arrangement used, whereas Figures 10, 11, 13 and 14 show the results of the simulations.

The different source and receiver arrangements were elaborated in order to better visualize the phenomena associated with the propagation of elastic waves in porous media, so these arrangements may not be feasible in practical applications and experiments, being fundamentally used to better understand the results.

In addition, the graphs of the solutions were selected until a certain time to show the main seismic events indicated by the arrows, but the events continue to appear after this time, with less amplitude, due to the dispersion and attenuation of the waves.

Figure 10 shows the results obtained for the lowfrequency simulated case, where the absolute displacement velocity of the matrix was plotted for both the Biot model (blue curve) and the Biot-JKD model (red curve). The numbers in the graph indicate the arrival times of the events detected in this simulation. Due to the fact that a source has been used in the low frequency domain, all events indicated are related to the propagation of the fast $\mathrm{P}$ wave in the poroelastic medium, there is no slow $P$ wave. Indicative arrow number 1 refers to the direct wave emitted by the source and captured by the receiver, while number 2 indicates the reflected wave in the first interface $z_{1}$, arrow number 3 is related to the reflection in the second interface $z_{2}$, already the number 4 is associated with the first internal multiple and the number 5 to the second internal multiple. Figure 11 shows the same result as Figure 10, but with a zoom in the ordinate axis, to improve the visualization of smaller amplitude events.

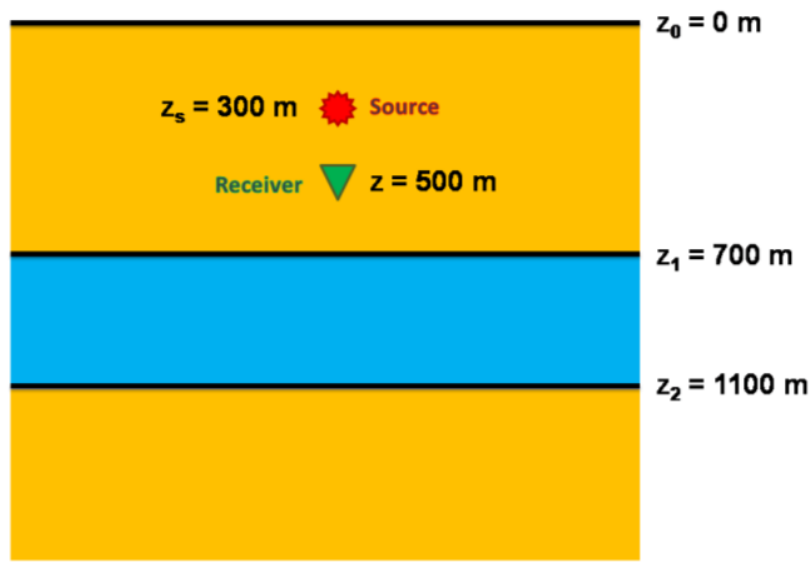

Figure 9: Geological model and source-receiver arrangement used for low frequency case simulation
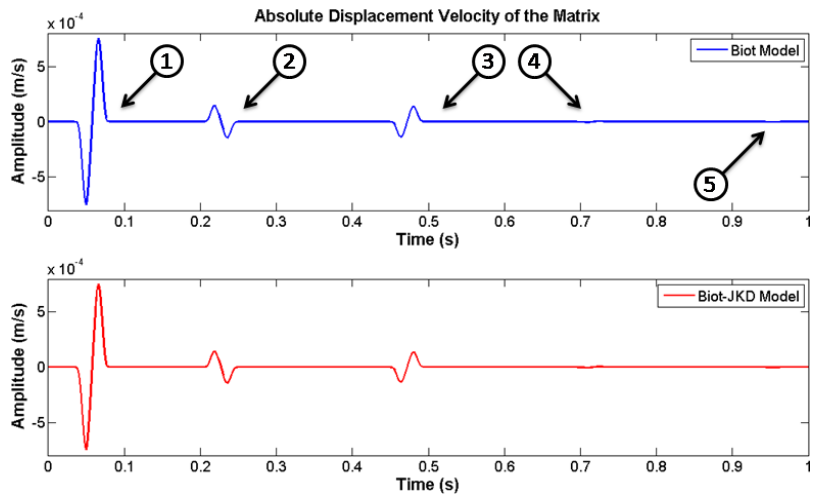

Figure 10: Absolute displacement velocity of the matrix for low frequency case simulation
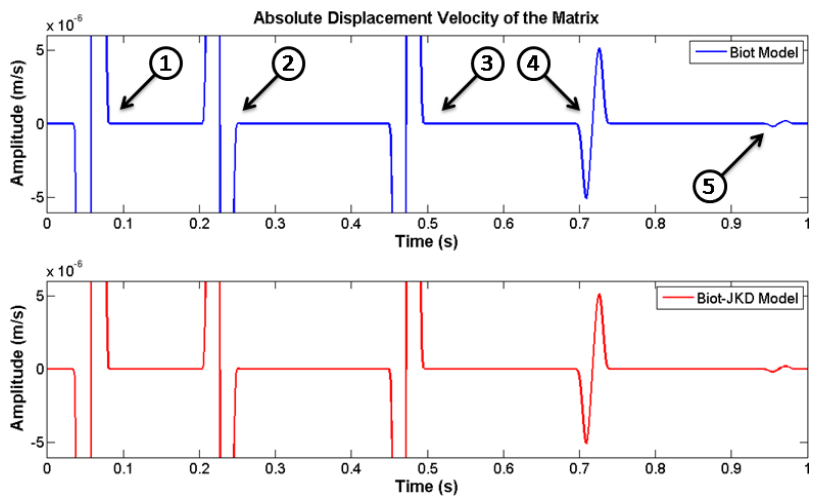

Figure 11: Absolute displacement velocity of the matrix for low frequency case simulation with zoom in $\mathrm{Y}$-axis

Figures 13 and 14 show the result for the simulated case of high frequencies. Arrow number 1 indicates the direct fast wave, number 2 points to the fast wave reflected in the first interface $z_{1}$, arrow 3 indicates the fast wave that was converted to slow when reflecting in the interface $z_{1}$, 
the numbering 4 is referring to the slow wave direct event, event 5 is associated with the reflection of the fast wave at the second interface $z_{2}$, the fast wave reflected at the second interface $z_{2}$ and converted to slow when refracted, on its return path, at the first interface $z_{1}$ is indicated by the arrow 6, the numbering 7 points to the event determined by the slow wave converted to fast as it reflects on the first interface, while arrow 8 signals the slow wave reflected in the first interface $z_{1}$ and the ninth indication refers to the first internal multiple of the fast wave.

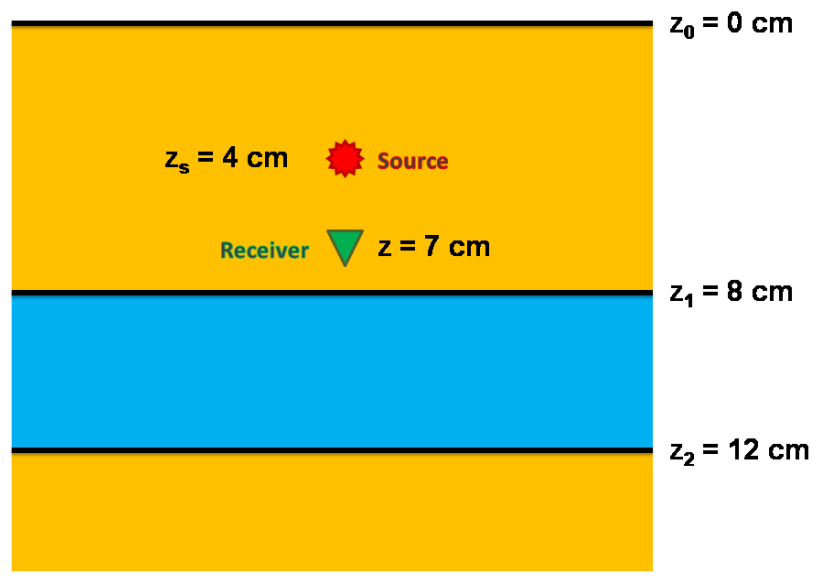

Figure 12: Geological model and source-receiver arrangement used for high frequency case simulation
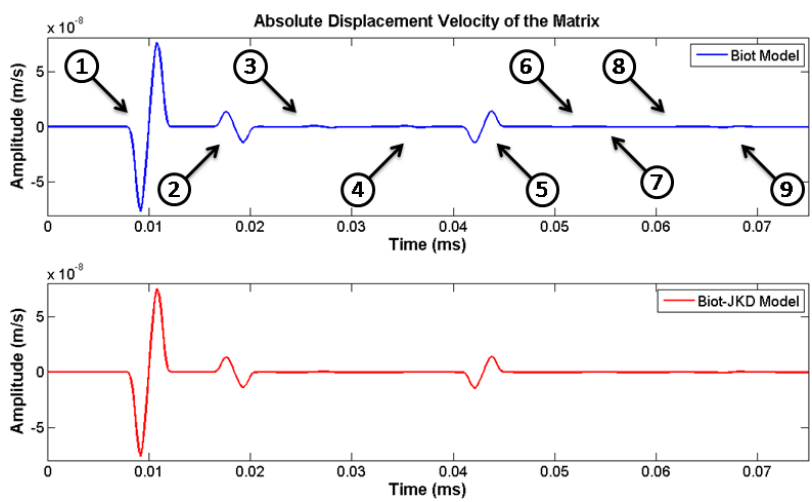

Figure 13: Absolute displacement velocity of the matrix for high frequency case simulation
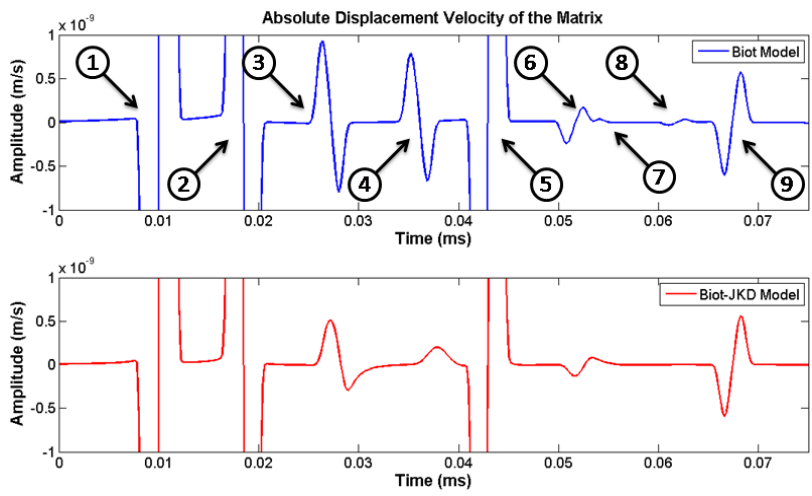

Figure 14: Absolute displacement velocity of the matrix for high frequency case simulation with zoom in $\mathrm{Y}$-axis
As the medium considered in the simulation is onedimensional (1D), there is no presence of the shear wave, and it is possible to visualize only the $P$ waves. It was observed that the result found for the domain of the high frequencies presented a difference of amplitude for the events related to the slow wave between the two models, Biot and Biot-JKD. The explanation for this phenomenon is found in the dispersion and attenuation analysis of the waves, where it can be observed that the attenuation for the Biot-JKD model is higher than for the Biot model. However, to low frequency case, there were no differences in amplitude between the Biot and Biot-JKD models, which was already expected theoretically, given that for this domain the dispersion curves are similar.

Another very important phenomenon that was observed in the simulation results for the high-frequency domain was the conversion of the fast-slow and also slow-fast compressional wave, where the elastic wave propagates in the poroelastic medium, converting its propagation mode by refracting or reflect at an interface between two distinct porous media, such phenomenon was also observed by Bouzidi (2013).

\section{Conclusions}

In this work, both the low frequencies and the high frequencies were considered, and it was assumed that the pores of the porous medium are interconnected and completely filled by fluids. The main objective was to simulate, considering a mixed problem for the systems of the Biot and Biot-JKD equations, the elastic response of a stratified 1D porous medium. In addition, a wave dispersion and attenuation analysis was performed, relating frequency dependence to phase velocities and wave attenuation. Four different porous media were considered: saturated with water, light oil, medium oil and heavy oil, comparing the dispersion curves for both the Biot and Biot-JKD models and the influence of the different types of fluid that saturate the pores of the rock. For low frequencies and frequencies well above the critical frequency, it was observed that denser the fluid, lower the velocity of the fast wave phase. Moreover, the more viscous the fluid, the slower the attenuation of the slow wave, both for the Biot model and the Biot-JKD model and for low frequencies, the more viscous the fluid, the lower the wave attenuation; for very high frequencies, the opposite occurs. In the results obtained through the simulations, it was possible to confirm the fundamentals of the poroelasticity theory. As seen throughout this work, the slow wave propagation of Biot does not occur at low frequencies, only the fast wave, which was proved by the seismic response. For the high frequency domain, the inertial effects are predominant and the slow mode is activated, in this way, the slow compressional wave propagation occurs. In addition, during the simulations for the high-frequency domain, the presence of the fast-slow and also slow-fast converted $P$ wave was observed, where the elastic wave propagates converts its propagation mode by refracting or reflecting in an interface of discontinuity between two distinct poroelastic media. 


\section{Acknowledgments}

The authors thank the "Programa de Formação de Recursos Humanos da Petrobras", PRH-PB 226 (PETROBRAS/UENF) for the financial support and the "Laboratório de Engenharia e Exploração de Petróleo" (LENEP/CCT/UENF) for providing the necessary conditions for the development of this work.

\section{References}

AL-BESHARAH, J. M.; SALMAN, O. A.; AKASHAH, S. A. Viscosity of crude oil blends. Ind. eng. Chem. Res., n. 26, p. 2445-2449, 1987.

AZEREDO, M. M. Modelagem Matemática e Computacional da Propagação de Ondas Sísmicas em Meios Poroelásticos Estratificados. Tese (Doutorado) Universidade Estadual do Norte Fluminense Darcy Ribeiro, Maio 2013.

BIOT, M. A. Theory of propagation of elastic waves in a fluid-saturated porous solid. i. low-frequency range. The Journal of the Acoustical Society of America, v. 28, n. 2, p. 168-178, March 1956a.

BIOT, M. A. Theory of propagation of elastic waves in a fluid-saturated porous solid. ii. higher frequency range. The Journal of the Acoustical Society of America, v. 28, n. 2, p. 179-191, March 1956b.

BLANC, E. Time-Domain Numerical Modeling of Poroelastic Waves: The Biot-JKD Model with Fractional Derivatives. Tese (Doutorado) - AIX-Marseille Université, December 2013.

BOUZIDI, Y. Numerical models of converted slow p-wave modes in porous media. In: Poromechanics $V$ ASCE. [S.I.: s.n.], 2013.

CARCIONE, J. M. Wave Fields in Real Media: Wave Propagation in Anisotropic, Anelastic, Porous and Electromagnetic Media. Second. [S.I.]: Elsevier, 2007.

DARCY, H. Les fontaines publiques de la ville de dijon: exposition et application des principes à suivre et des formules à employer dans les questions de distribution d'eau. Victor Dalmont, 1856.

GARCíA, O. C. C. Modelagem Sísmica em Arenitos: Efeito da Dispersão da Velocidade e do Tipo de Fluido. Tese (Doutorado) - Pontifícia Universidade Católica do Rio de Janeiro, Maio 2008.

JOHNSON, D. L.; KOPLIK, J.; DASHEN, R. Theory of dynamic permeability and tortuosity in fluid-saturated porous media. J. Fluid Mech., v. 176, p. 379-402, 1987.

MIRANDA, M. R. S. T. Método Matricial em Modelagem Poroelástica: Modelo de Biot-JKD. Dissertação (Mestrado) - Universidade Estadual do Norte Fluminense Darcy Ribeiro, Agosto 2016.

OLIVEIRA, I. B. Modelagem de propagação das ondas elásticas em meios porosos 1D: modelos de Biot vs. BiotJKD. Dissertação (Mestrado). Universidade Estadual do Norte Fluminense Darcy Ribeiro, Fevereiro 2018.
TERZAGHI, K. von. Die berechnung des durchlässigkeitsziffer des tones aus dem verlauf der hydrodynamischen spannungserscheinungen. p. 105123, 1923.

URSIN, B. Review of elastic and eletromagnetic wave propagation in horizontally layered media. Geophysics, v. 48, n. 8, p. 1063-1081, August 1983. 